Dynamics and structural behavior of water in large confinement with planar amorphous walls

C. Gastón Ferrara and Tomás S. Grigera

Citation: The Journal of Chemical Physics 147, 024705 (2017); doi: 10.1063/1.4991834

View online: http://dx.doi.org/10.1063/1.4991834

View Table of Contents: http://aip.scitation.org/toc/jcp/147/2

Published by the American Institute of Physics

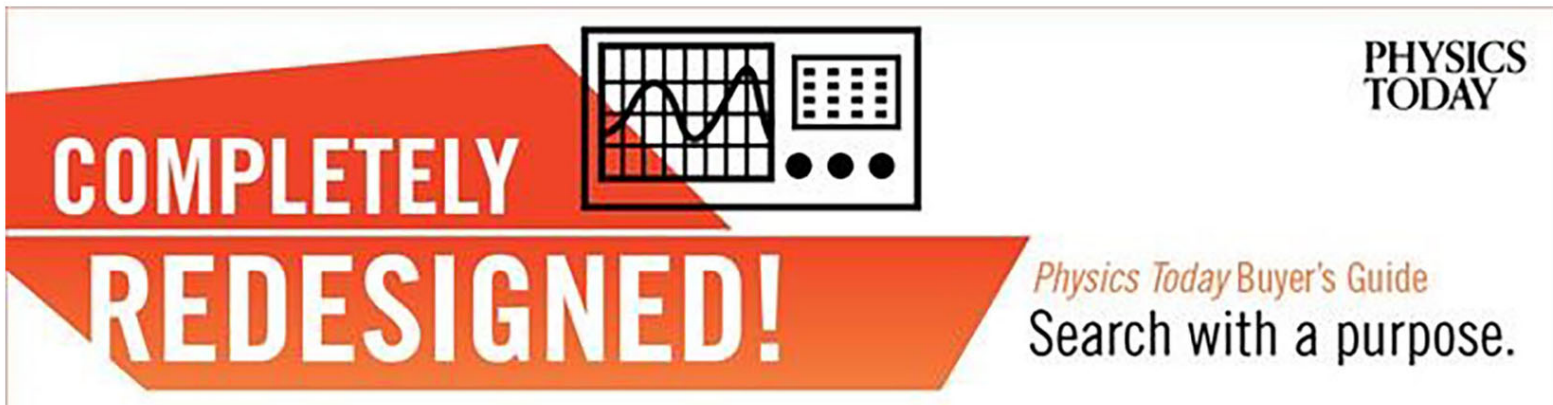




\title{
Dynamics and structural behavior of water in large confinement with planar amorphous walls
}

\author{
C. Gastón Ferrara ${ }^{1,2}$ and Tomás S. Grigera ${ }^{2,3,4}$ \\ ${ }^{1}$ Universidad Nacional Arturo Jauretche, Florencio Varela, Argentina \\ ${ }^{2}$ Instituto de Física de Líquidos y Sistemas Biológicos (IFLYSIB), CONICET and Facultad de Ciencias Exactas, \\ Universidad Nacional de La Plata, Calle 59 No. 789, B1900BTE La Plata, Argentina \\ ${ }^{3}$ CCT CONICET La Plata, Consejo Nacional de Investigaciones Científicas y Técnicas, La Plata, Argentina \\ ${ }^{4}$ Departamento de Física, Universidad Nacional de La Plata, C.C. 67, 1900 La Plata, Argentina
}

(Received 28 September 2016; accepted 23 June 2017; published online 13 July 2017)

\begin{abstract}
We study the structure and dynamics of liquid water confined between planar amorphous walls using molecular dynamics (MD) simulations. We report MD results for systems of more than $23000 \mathrm{SPC} / \mathrm{E}$ water molecules confined between two hydrophilic or hydrophobic walls, separated by distances of about $15 \mathrm{~nm}$. We find that the walls induce ordering of the liquid and slow down the dynamics, affecting the properties of the confined water up to distances of about $8 \mathrm{~nm}$ at $275 \mathrm{~K}$. We quantify this influence by computing dynamic and static penetration lengths and studying their temperature dependence. Our results indicate that in the temperature range considered, hydrophobic walls perturb static properties over larger lengths compared to hydrophilic walls. We also find opposite temperature trends in the dynamic penetration lengths, with hydrophobic walls increasing their range of influence on increasing the temperature. Published by AIP Publishing. [http://dx.doi.org/10.1063/1.4991834]
\end{abstract}

\section{INTRODUCTION}

The role of water in almost all aspects of life has made it an object of intense investigation. It is now clear that its structural and dynamical properties make it a liquid that stands out from the rest, and its properties make it play a central role in different disciplines, from nanofluidics to biology. ${ }^{1}$ The last few years have witnessed a large number of experimental and numerical studies of confined water, both in nanopores ${ }^{2-7}$ and between planer walls (smooth, crystalline, and amorphous), ${ }^{8-13}$ seeking to shed light on the changes that take place in its properties compared to bulk water.

Most of these works have focused on the mechanisms of freezing. It has been observed that phase transitions are strongly affected by the geometric and structural characteristics of the confinement. In general, a drop in the freezing temperature has been found. The role of the walls (hydrophobic or hydrophilic) seems crucial, since very small tubes make it experimentally impossible to observe crystallization. ${ }^{14}$ Recent experimental evidence shows that ice-like structures form at room temperature under nanoconfinement. ${ }^{15}$ It has also been observed that in nanopores, there are different layers, with water near a hydrophilic surface suffering an important slow down at room temperature. ${ }^{16}$

In this work, we considered water confined between planar amorphous walls, separated by a distance much larger than that in the majority of previous studies. ${ }^{17-20} \mathrm{We}$ considered both hydrophilic (PHY) and hydrophobic (PHO) walls, aiming to study the changes that it induces in the confined water. We choose amorphous walls to avoid ordering induced by the periodicity of the walls and a large wall separation to focus on the changes induced by the interaction rather than on small-size effects.
We find that the walls perturb the structure and dynamics of water up to distances of $8 \mathrm{~nm}$ or more, and that the effect of $\mathrm{PHO}$ and PHY walls has a qualitatively different temperature dependence.

\section{SYSTEM AND SIMULATION DETAILS}

\section{A. System}

We consider a collection of SPC/E water molecules ${ }^{21}$ confined by two parallel amorphous walls (a "sandwich" geometry). We have studied two kinds of wall: hydrophilic (made of restrained water molecules) and hydrophobic (made of glassy Lennard-Jones particles). The simulation box was $4 \mathrm{~nm} \times 4 \mathrm{~nm} \times L$, with $L$ between $33 \mathrm{~nm}$ and $35 \mathrm{~nm}$ (fluctuating due to coupling with a barostat). We choose the $x y$ plane parallel to the walls and the $z$ axis to measure the distance from the wall. Periodic boundary conditions are employed in all axes (so actually there is only one wall but wider than the water-water interaction cutoff so that the water molecules do not feel the presence of water beyond the wall).

The hydrophilic wall was made from an equilibrated bulk water system by harmonically restraining the oxygens of all water molecules within a $2.8 \mathrm{~nm}$ wide slab (containing 2300 molecules) perpendicular to the $z$ axis. The total size was $4 \mathrm{~nm} \times 4 \mathrm{~nm} \times 35 \mathrm{~nm}$, with 23333 mobile molecules confined.

The hydrophobic wall was built using a Lennard-Jones binary mixture (similar to the Kob-Andersen system ${ }^{22}$ ), with parameters chosen so as to achieve a glass (mode-coupling) transition temperature of around $700 \mathrm{~K}$. Then a slice of the bulk system was cleared of water molecules and filled with the Lennard-Jones particles. This resulted in a hydrophobic 


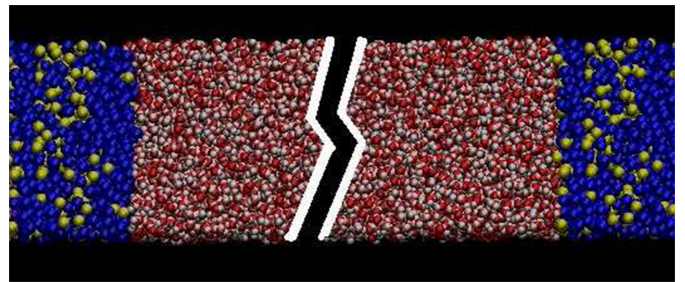

FIG. 1. Snapshot of the water system confined with hydrophobic wall. Water molecules are painted in red and white, and the components of the hydrophobic wall are blue and yellow.

wall of $3 \mathrm{~nm}$ thick in a box of size $4 \mathrm{~nm} \times 4 \mathrm{~nm} \times 33 \mathrm{~nm}$, with 22226 confined water molecules (Fig. 1).

\section{B. Simulations}

Molecular dynamics (MD) simulations in the NpT ensemble were done with GROMACS. ${ }^{23}$ Temperature and pressure were controlled with a Berendsen thermostat and a Berendsen barostat, ${ }^{24}$ keeping the isotropic hydrostatic bath at 1 bar. Long-range interactions were computed using the reaction field method ${ }^{25}$ with a dielectric constant $\epsilon=78$ and a cutoff $1.4 \mathrm{~nm}$, and the integration step used during stabilization was $2 \mathrm{fs}$. We used all-atoms force field ffgmx 2 for the minimization process, as well as for all the MD simulations. ${ }^{26-30}$

A precursor system of bulk SPC/E water (25 114 molecules) was first equilibrated for $460 \mathrm{~ns}$ at $300 \mathrm{~K}$ and 1 bar. An annealing procedure was then used to obtain bulk systems at $315 \mathrm{~K}, 285 \mathrm{~K}, 280 \mathrm{~K}$, and $275 \mathrm{~K}$ (at the same pressure). After reaching the final temperature, each system was further stabilized for another $100 \mathrm{~ns}$.

The hydrophilic wall was built from the precursor system at $300 \mathrm{~K}$, using a harmonic constant of $1000 \mathrm{~kJ} / \mathrm{mol} \mathrm{nm}{ }^{2}$ to restrain the free movement of 1809 oxygenous, forming a wall of the order of $3 \mathrm{~nm}$ thick. This system was then stabilized for $160 \mathrm{~ns}$; annealing was again used to obtain confined systems at the other listed temperatures.

For the hydrophobic walls, the L-J binary mixture was equilibrated at $800 \mathrm{~K}$ and then suddenly quenched to $300 \mathrm{~K}$. A slice of the precursor system was then cleared of water molecules and filled with the Lennard-Jones particles. The LJ particles interaction with the water oxygens is also LennardJones, with an energy scale $\epsilon_{A}=\epsilon_{B}=0.65017 \mathrm{~kJ} / \mathrm{mol}$ (identical to that used for the oxygen-oxygen interaction), and with diameters $\sigma_{A}=0.4 \mathrm{~nm}$ and $\sigma_{B}=0.35 \mathrm{~nm}$. The confined system was stabilized at $300 \mathrm{~K}$ for $430 \mathrm{~ns}$, and then annealing was used to obtain different temperatures. We used five different realizations of the amorphous wall; all results reported are averaged over the five realizations.

After stabilization, for each temperature and wall type, four different equilibrium configurations were chosen and simulated for another $1 \mathrm{~ns}$, collecting data every $0.2 \mathrm{ps}$.

\section{Measured quantities}

We studied structural and dynamical quantities of water as a function of the distance $z$ from the edge of the confining wall, averaging over narrow slices parallel to it. Structure was characterized through the local density and the distribution of hydrogen bonds (HBs). The density is computed as $\rho=\mathrm{Nm} / \mathrm{V}$, where $m$ is the mass of a water molecule and $N$ is the number of molecules in the given volume $V$. To compute the local density, the volume was discretized in cubes of side $0.1 \mathrm{~nm}$.

The number of HBs was computed using a geometric definition, ${ }^{31}$ according to which a hydrogen bond exists between two water molecules whenever the distance between oxygen atoms is less than $0.35 \mathrm{~nm}$ (minimum of RDF for SPC/E), and the angle formed by the vector $\mathrm{X}$ (oxygen-oxygen) and the $\mathrm{O}-\mathrm{H}$ vector of the $\mathrm{H}$-donor is less than $30^{\circ}$. The distribution of the number of HBs per water molecules as a function of distance from the wall was calculated and averaged for all the water molecules present in $0.1 \mathrm{~nm}$ slabs parallel to the surfaces.

To analyze the local dynamics, we use the self-overlap or coarse-grained density autocorrelation. ${ }^{32}$ We partition the simulation box in many small cubic boxes of side $\ell=0.1 \mathrm{~nm}$, such that the probability of finding more than one particle in a single box is negligible. If $n_{i}$ is the number of particles in box $i$, then

$$
q(z, t)=\frac{1}{\ell^{3} N_{i}} \sum_{i \in v}\left\langle n_{i}(0) n_{i}(t)\right\rangle,
$$

where the sum runs over all boxes that lie on slice centered at $z, N_{i}$ is the number of boxes in the slice, and $\langle\ldots\rangle$ indicates a thermal average. A cube is considered occupied by a water molecule if the oxygen lies within the cube. Normalization is such that the overlap of two identical configurations is 1 on average, while for totally uncorrelated configurations $q_{=} q_{0}$ $\equiv \ell^{3}=10^{-3}$.

\section{RESULTS}

\section{A. Structure}

\section{Density profile}

We have computed the density as a function of $z$ (the direction perpendicular to the walls), averaged over $0.3 \mathrm{~nm}$ slices. The confining walls perturb the structure of the nearby water molecules, with a range that depends on the characteristics of the wall and the temperature. Figure 2 shows the (mass) density of water and wall molecules in the region of the interface, where the water density grows monotonically from zero to a maximum value which is above the bulk value for the same temperature (see also Table I). The origin $z=0$ is defined by the first bin where a water molecule is found. The width of

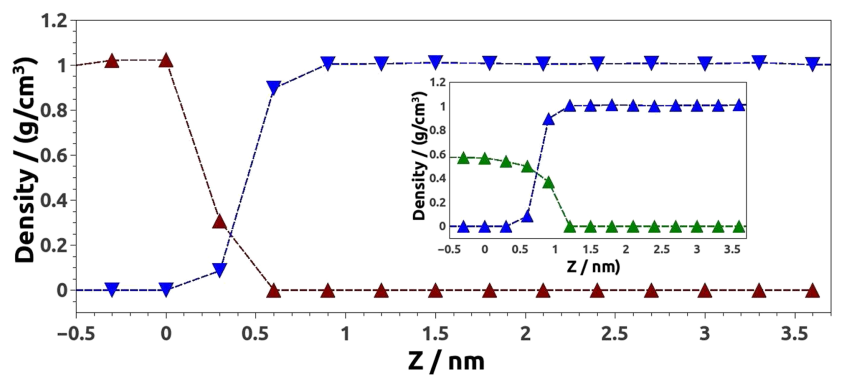

FIG. 2. Density vs. distance from the wall at $T=300 \mathrm{~K}$ in the region of the interface between water (blue triangles) and the PHY wall (brown triangles), PHO wall (green triangles), and bulk (blue triangles). $z=0$ is defined as the first bin where water molecules are detected. 
TABLE I. Maximum density reached near the wall $\left(\mathrm{g} / \mathrm{cm}^{3}\right) . \Delta \rho$ is the difference between the maximum and the bulk.

\begin{tabular}{llcccc}
\hline \hline $\mathrm{T}(\mathrm{K})$ & \multicolumn{1}{c}{ Bulk } & PHO & PHY & $\Delta \rho$ PHO & $\Delta \rho$ PHY \\
\hline 275 & $1.00757(8)$ & $1.015(2)$ & $1.012(3)$ & 0.008 & 0.005 \\
280 & $1.00554(5)$ & $1.009(1)$ & $1.011(2)$ & 0.004 & 0.006 \\
285 & $1.00368(8)$ & $1.017(1)$ & $1.012(1)$ & 0.014 & 0.009 \\
300 & $0.9965(1)$ & $1.006(3)$ & $1.006(4)$ & 0.010 & 0.010 \\
315 & $0.98780(6)$ & $1.001(3)$ & $1.004(3)$ & 0.014 & 0.017 \\
\hline
\end{tabular}

the interface region is the same for both PHY and PHO walls (about $1 \mathrm{~nm}$ ), although with the PHO wall the initial slope is slightly higher. The value difference between the maximum density and the bulk density increases with temperature for the PHY wall, while there is no systematic trend in the PHO case (Table I).

Away from the interfacial region (i.e., after the maximum is reached), the density shows both short wavelength oscillations and a slow decay (although its amplitude is very small, about $1 \%$ of the bulk density). Figures 3 and 4 show this decay together with an exponential fit,

$$
\rho(z)=\left(1-\rho_{0}\right) e^{-z / \xi_{s}}+\rho_{0},
$$

where the fit is done over half the wall-to-wall distance.

The penetration length $\xi_{s}$ is a measure of the range of the perturbation introduced by the confining wall. We find that the PHO wall perturbs the density to larger scales than the PHY

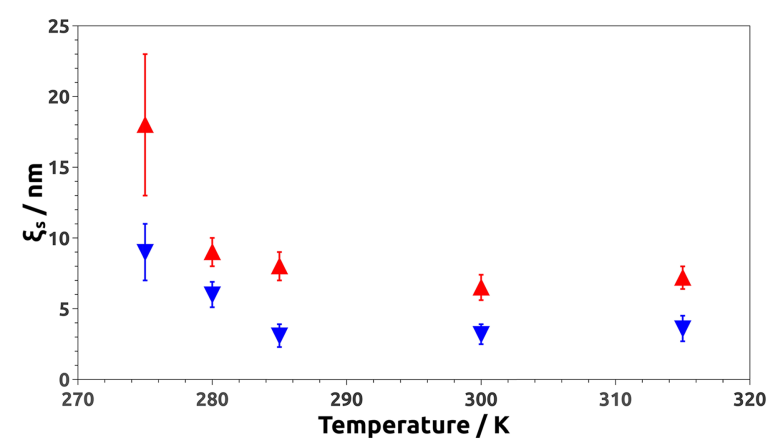

FIG. 5. Static penetration length $\xi_{s}$ as a function of temperature for both walls (red triangles facing upwards: PHO, blue triangles facing downwards: PHY).

wall at all temperatures (Fig. 5). For both walls, the value of $\xi$ decreases with rising temperature; in this sense, the effect of both walls weakens with temperature.

In the PHO case, the bulk density is never reached for temperatures less than $315 \mathrm{~K}$, while in the PHY wall the density reaches a value smaller than the corresponding bulk density. This is similar to what has been found in other simulations of confined water (e.g., Refs. 18 and 33). The values of the penetration lengths are rather large, but we emphasize that this applies to the rather small decay (of the order of $1 \%$ ) of density beyond the first maximum near the wall. At a scale suitable to observe the increase of density near the wall (such as Fig. 2), this decay is not discernible.
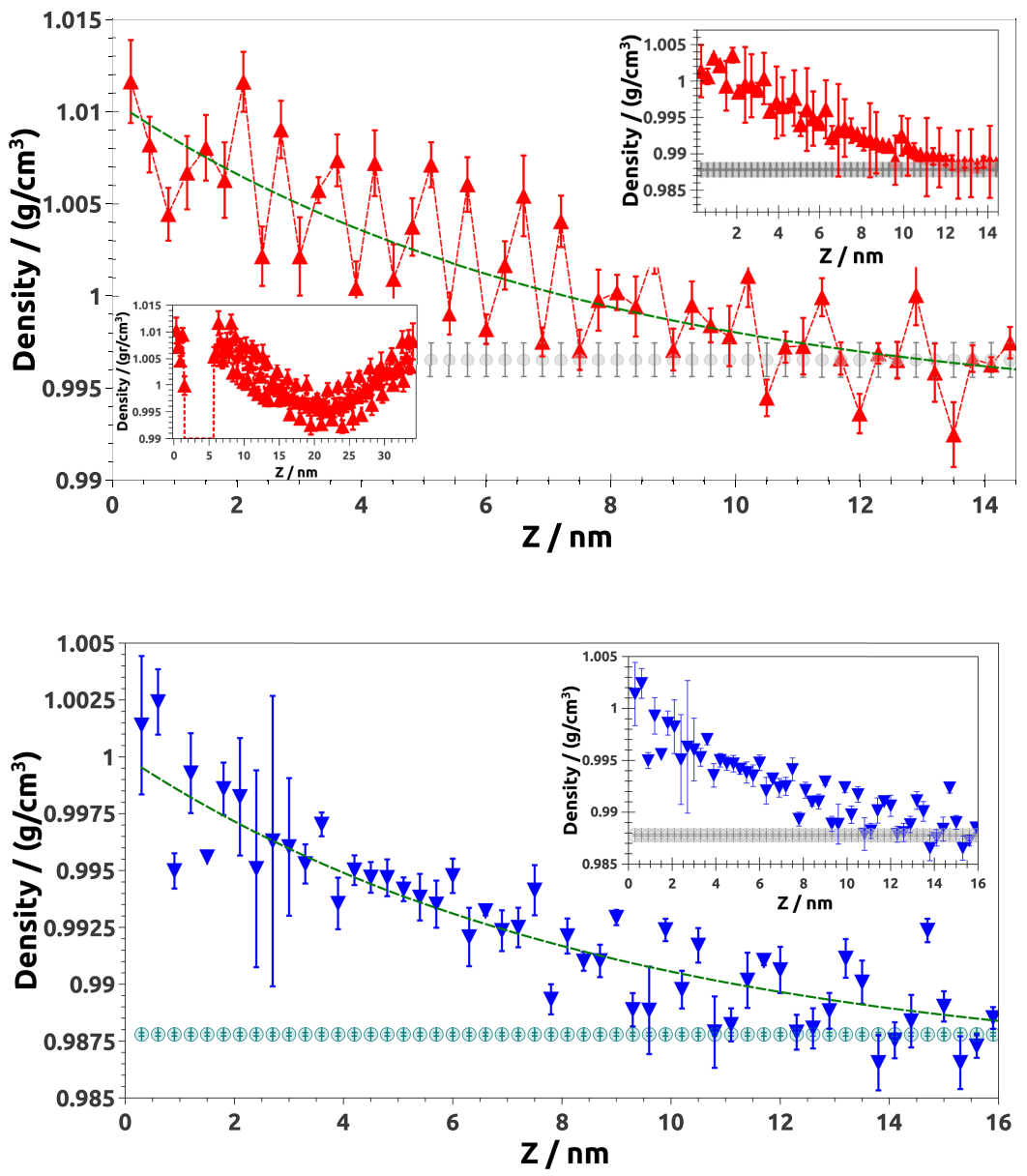

FIG. 3. Decay of water density vs. $z$ (distance from wall) for the PHO wall at $T=300 \mathrm{~K}(T=315 \mathrm{~K}$ in the top right inset), for $z$ beyond the first maximum of the density and up to the point halfway between the two periodic images of the wall. The bulk density is shown in grey and the continuous line is the exponential fit [Eq. (2)]. Bottom left inset: water density for the full simulation box, showing the position of the PHO wall and the increase in density as the positions beyond the half of the box approach the periodic image of the wall from the opposite side. In the inset, the origin of the $z$ coordinate is the same as in the actual simulations and is shifted with respect to the $z$ coordinate used elsewhere in the article.

FIG. 4. Same as Fig. 3 but for the PHY wall at $T=300 \mathrm{~K}$ and $T=315 \mathrm{~K}$ (inset). 


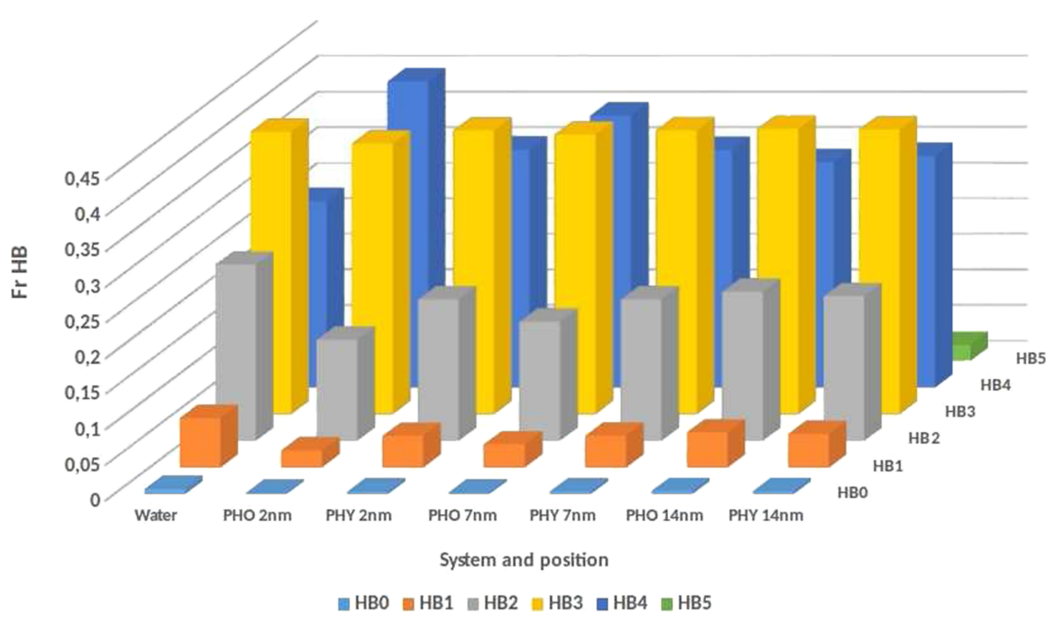

FIG. 6. Histograms of the number of hydrogen bonds per water molecule for both kinds of walls at $300 \mathrm{~K}$ and $z=2 \mathrm{~nm}, 7 \mathrm{~nm}$, and $14 \mathrm{~nm}$. Bulk values are shown in the first row.

\section{Hydrogen bond distribution}

We now consider the distribution of the number of HBs per water molecule as a function of the distance from the wall (HBs were computed with a geometrical criterion as explained in $\mathrm{Sec}$. II C). Figure 6 shows histograms of the number of HBs at selected distances from the wall at $T=275 \mathrm{~K}$. The histograms show that it is the number of water molecules with 2 and $4 \mathrm{HBs}$ (called HB2 and HB4) that is most affected by the presence of the wall so that we study these in more detail. Figures 7 and 8 (for $T=275 \mathrm{~K}$ ) and Figs. 9 and $10(T=300 \mathrm{~K}$ ) show the fraction of HB2 and HB4 water molecules as a function of $z$. We find that the fraction of water molecules forming $2 \mathrm{HBs}$

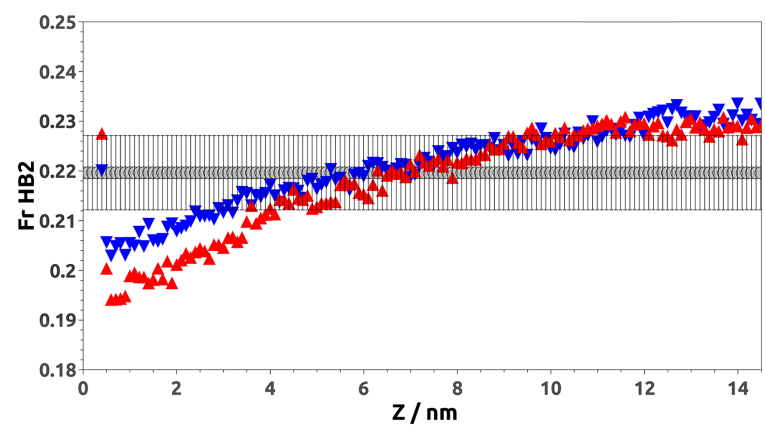

FIG. 7. Fraction of HB2 water molecules as a function of distance from the wall at $T=275 \mathrm{~K}$. Red triangles facing upwards: PHO wall, blue triangles facing downwards: PHY wall, grey circles: bulk.

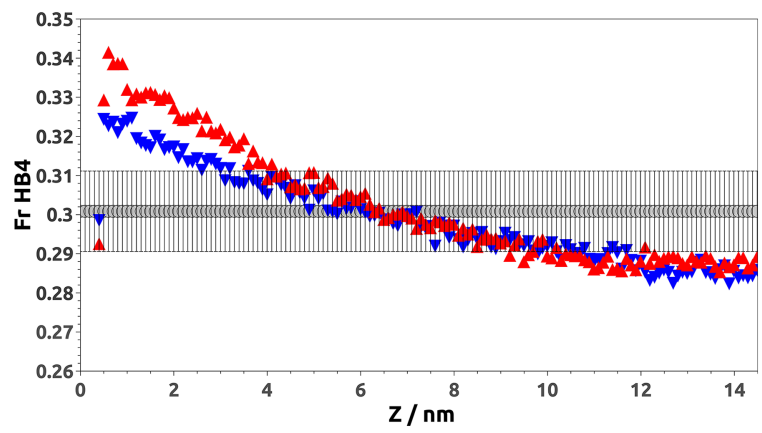

FIG. 8. Fraction of HB4 water molecules as a function of the distance from the wall at $T=275 \mathrm{~K}$. Red triangles facing upwards: $\mathrm{PHO}$ wall, blue triangles facing downwards: PHY wall, grey circles: bulk. is reduced near the walls, while correspondingly the fraction forming 4 bonds increases. This happens for both kinds of wall and at all the temperatures we studied, but is more pronounced for the PHO case, and the strength of the effect decreases with rising temperature. Around 6-8 nm, the distribution of $\mathrm{HBs}$ is similar to that of bulk water, but further from the wall it deviates again, although in the opposite direction. This corresponds to the confined system crossing the bulk density at $6-8 \mathrm{~nm}$ (Fig. 6).

The absolute number of HB4 molecules (not shown) tracks closely the variation in density, but the number of HB2 molecules shows the opposite behavior. Since we have used a geometric criterion to count HBs, one would expect that a drop in density be correlated with a drop in the number of HBs

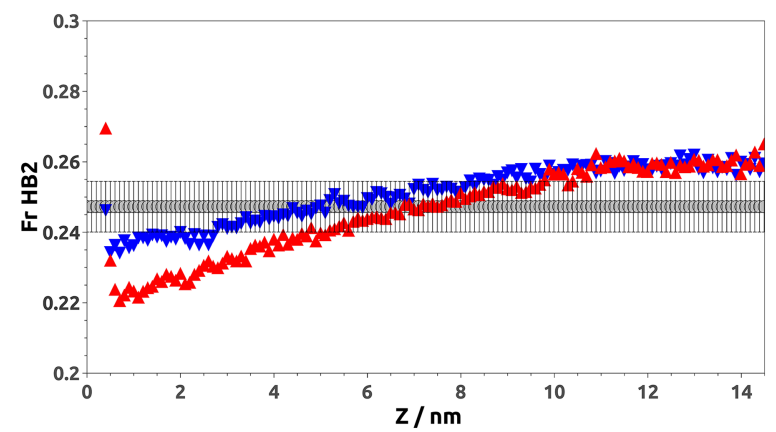

FIG. 9. Fraction of HB2 water molecules as a function of the distance from the wall at $T=300 \mathrm{~K}$. Red triangles facing upwards: $\mathrm{PHO}$ wall, blue triangles facing downwards: PHY wall, grey circles: bulk.

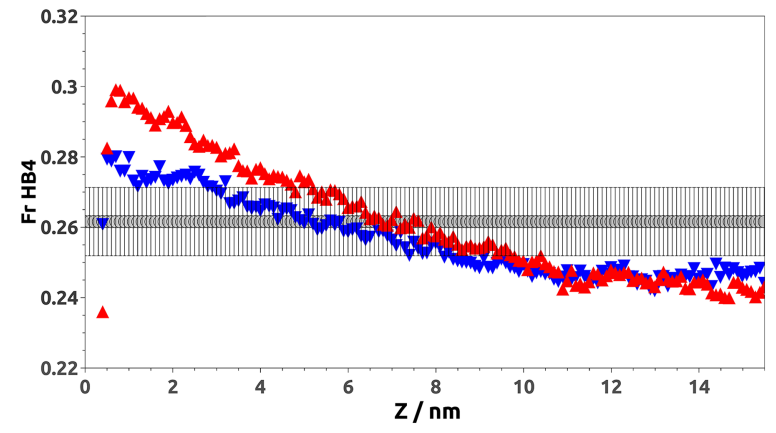

FIG. 10. Fraction of HB4 water molecules as a function of the distance from the wall at $T=300 \mathrm{~K}$. Red triangles facing upwards: $\mathrm{PHO}$ wall, blue triangles facing downwards: PHY wall, grey circles: bulk. 


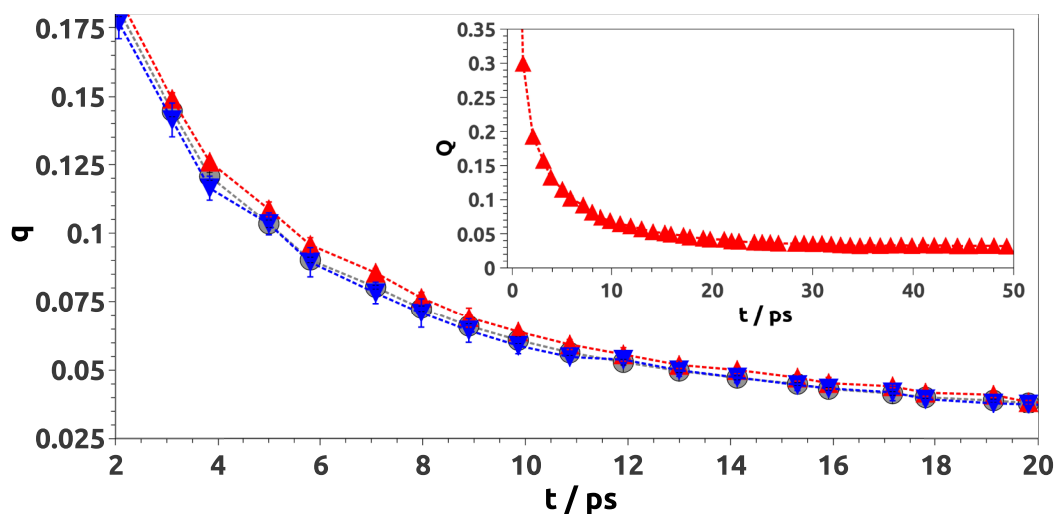

FIG. 11. Density self-correlation function (overlap) vs. time at $z=6.9 \mathrm{~nm}$ (red triangles facing upwards: $\mathrm{PHO}$ wall, blue triangles facing downwards, PHY wall, grey circles: bulk). The gray circles are not seen in the figure by overlapping with the figures of the PHO and PHY walls. Inset: overlap vs time at $z=13.5 \mathrm{~nm}$ (PHO wall) together with a stretched exponential fit. $T=275 \mathrm{~K}$.

as the mean interparticle distance grows. However, we record a change in the distribution of the number of molecules with different amounts of HBs, affecting mainly the proportion of HB2 and HB4, while the proportion of HB3 and HB1 remains virtually unchanged. This suggests instead a subtle change in structure. These results, together with the density, show that the presence of a wall perturbs the structure up to distances larger than previously thought. ${ }^{34,35}$ The wall induces an ordering of the water, stronger for lower temperatures and PHO interactions, as evidenced by the increase of the HB4 waters. However, this order is not related to order within the wall itself, since both walls are amorphous. It is also clear that the structure is not ice-like, as the density increases together with the ordering.

\section{B. Dynamics}

To probe the dynamics, we have computed the timedependent density overlap (Sec. II C) as a function of $z$, in slices $0.3 \mathrm{~nm}$ wide. For example, Fig. 11 shows the time decay of the overlap for the bulk liquid as well as for the confined water at a fixed position. We find that a simple exponential does not adequately fit the time decay since it has a long tail. Instead, a good fit is obtained with a stretched exponential (Kolrausch-William-Watts relaxation ${ }^{36,37}$ ),

$$
q(z, t)=A e^{-(t / \tau(z))^{\beta(z)}}+q_{0},
$$

where $\tau(z)$ is a time scale, $\beta(z)$ is called the stretching exponent (with smaller $\beta$ giving a longer tail), and $q_{0}$ and $A$ are fit parameters that depend on temperature. The inset of Fig. 11 shows the stretched exponential fit for a slice $13.5 \mathrm{~nm}$ from the PHO wall at $T=275 \mathrm{~K}$. A stretched exponential decay for a dynamical correlation has also been found, e.g., for water in silica nanopores, ${ }^{38}$ although with higher values of $\beta$.

The stretched exponential can be interpreted as the result of many exponential relaxation processes, with a wide distribution of relaxation times, ${ }^{36}$ with smaller $\beta$ indicating a wider distribution. We find that $\beta$ and $\tau$ show opposite trends as a function of $z$, with the characteristic time $\tau$ decreasing farther from the wall (apart from the interfacial region) but with $\beta$ increasing, signaling a narrowing of the distribution (see Figs. 12 and 13). This means that the dynamics speeds up away from the wall (an effect more pronounced for PHO walls), an interpretation confirmed by considering the average relaxation time, given by ${ }^{36}$

$$
\langle\tau(z)\rangle=\frac{\tau(z)}{\beta(z)} \Gamma\left(\frac{1}{\beta(z)}\right),
$$

where $\Gamma(x)$ is Euler's Gamma function.

The average relaxation time vs. $z$ for both walls and the respective fit at $T=275 \mathrm{~K}$ is shown in Fig. 14. It is seen to increase rapidly in the interfacial region over which the water density grows. This region is $1 \mathrm{~nm}$ wide for the PHY wall and $1.5-2 \mathrm{~nm}$ wide in the PHO case. Farther from the wall, $\langle\tau\rangle$ decays gradually towards the bulk value, reaching it only for distances larger than $8 \mathrm{~nm}$ (Fig. 14). Outside the region of the interface, the spatial decay was fit with an exponential

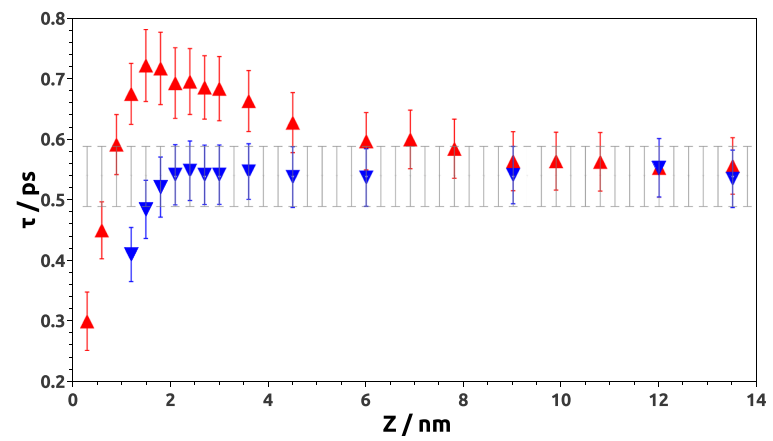

FIG. 12. Relaxation time vs. distance from the wall (red triangles facing upwards: PHO wall, blue triangles facing downwards: PHY wall, grey: bulk). The system were weakly coupled to a thermal and hydrostatic bath at $\mathrm{T}=275 \mathrm{~K}$ and $\mathrm{P}=1 \mathrm{bar}$.

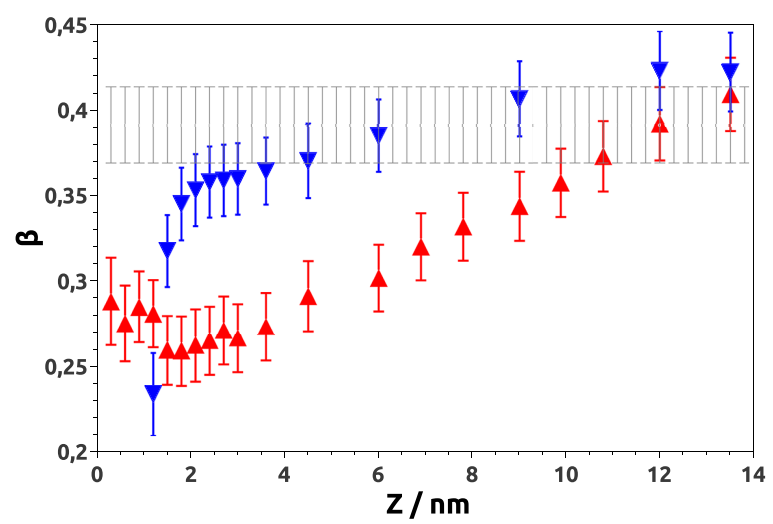

FIG. 13. The stretching exponent in terms of $\mathrm{z}$ position for both walls. ( $\triangle$ red) PHO, ( $\nabla$ blue) PHY, and ( $\mid$ grey) bulk. The system were weakly coupled to a thermal and hydrostatic bath at $\mathrm{T}=275 \mathrm{~K}$ and $\mathrm{P}=1$ bar. 


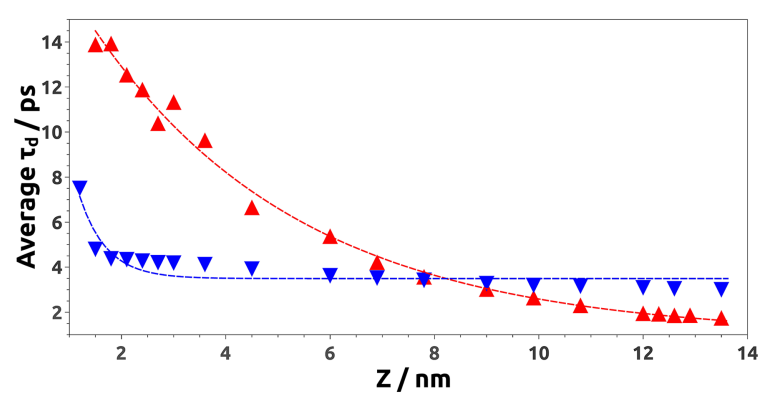

FIG. 14. Average relaxation time $\left\langle\tau_{d}\right\rangle$ vs $z$ for PHO wall (red triangles facing upwards) and PHY wall (blue triangles facing downwards) at $275 \mathrm{~K}$ and 1 bar. The fit is done only for the decaying region of the relaxation time, omitting the first few slices near the wall (not shown) where $\left\langle\tau_{d}\right\rangle$ has a sharp increase.

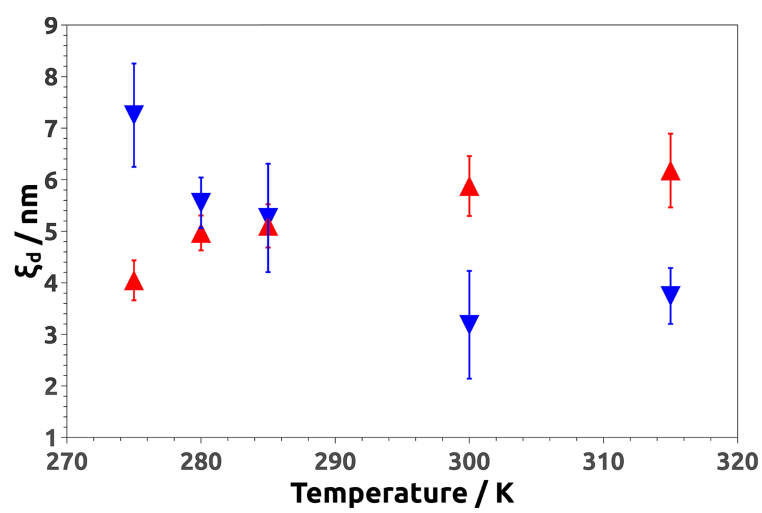

FIG. 15. Dynamic penetration length as a function of temperature for both walls. Red triangles facing upwards: PHO wall, blue triangles facing downwards: PHY wall.

decay,

$$
\langle\tau(z)\rangle=B e^{-z / \xi_{d}}+\tau_{0},
$$

where $\xi_{d}$ is the dynamic correlation length (the three fit parameters are temperature-dependent). $\xi_{d}(T)$ is a measure of the spatial extent of the influence of the wall on the dynamics; it is shown in Fig. 15.

The presence of both walls tends to slow down the dynamics near the wall, and the value of the dynamic correlation length is of the same order of magnitude for both walls in this range of temperature. However, the behavior of the correlation length with temperature is qualitatively different: while for PHY walls, our data are compatible with $\xi_{d}$ that stays constant or slightly decreases with temperature, for PHO walls, $\xi_{d}$ increases when the temperature is raised.

\section{SUMMARY AND CONCLUSIONS}

We have presented a study of the influence of confining amorphous walls on the dynamics and structure of water, in the case of relatively large distance between the walls. We have considered hydrophilic and hydrophobic walls, but in both cases we have chosen them to be amorphous, so as to avoid ordering effects induced by the wall structure. Since the PHY wall was made with restrained water molecules, this makes our study similar to that of Shekhar et al. ${ }^{39}$ in which a Lennard-Jones fluid is confined with Lennard-Jones walls. However, there are two crucial differences: our wall is pinned with a harmonic potential, not completely frozen, and we do not include a hard wall to strictly separate the wall and fluid. As a consequence, we can expect in the present case structural changes in the fluid which must be absent in the approach of Ref. 39.

We have found that the presence of the wall modifies both the structure and dynamics of water. We have observed, beyond a narrow $(\sim 1 \mathrm{~nm})$ interface region, a weak but relatively long range perturbation in the density profile (up to distances as large as $9 \mathrm{~nm}$ at $275 \mathrm{~K}$ ). The perturbation range can be characterized by an exponential penetration length $\xi_{s}$ [Eq. (2)], slightly larger for PHO walls. This range drops rapidly as temperature is raised (Fig. 5).

Density perturbations have been studied in confined situations before (e.g., Refs. 40 and 41), although here we are considering much larger separation between the walls. Compared to the more confined case, the perturbation we find is quantitatively smaller but of longer range. Near the center of the box, a density is reached lower than the bulk value, consistent with the experimental results of Erko et al. ${ }^{3}$

The distribution of hydrogen bonds shows that the structure near the walls is different from that of bulk water, with more molecules forming four HBs in detriment of molecules forming only two bonds. Beyond this region, water gradually loses structure (a less fraction of molecules forms four HBs) as the density drops. Again the effect is similar for both walls, but more pronounced in the PHO case. Raising the temperature reduces the structural changes brought in by the walls.

The dynamical influence of the walls was studied through the overlap (density self-correlation function). As in the structural case, we found that both walls have a similar effect, bringing in a slow down near the wall (except for the first few low-density layers), as well as a more heterogeneous (wider
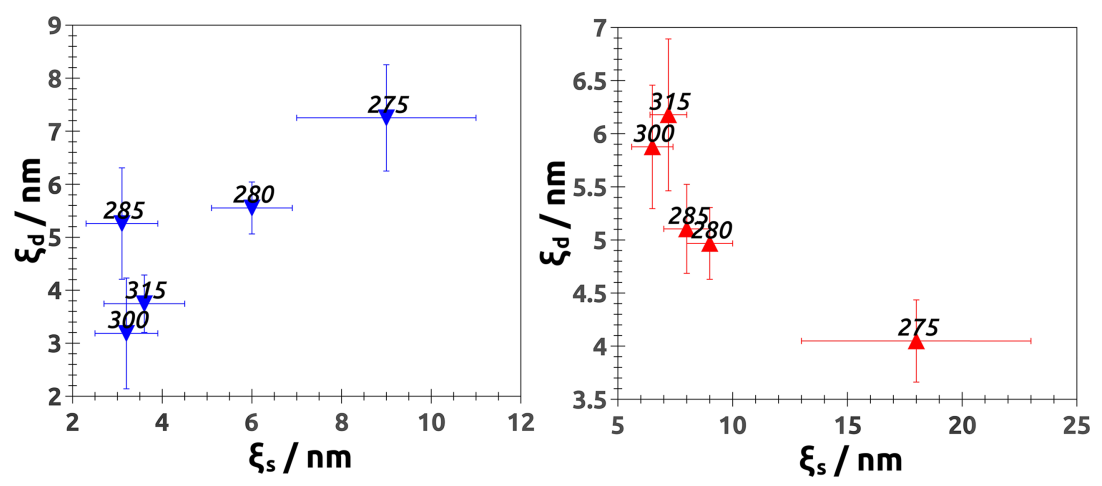

FIG. 16. Dynamic correlation length $\xi_{d}$ vs. static penetration length $\xi_{s}$ for the PHY (left) and PHO (right) walls. 
distribution of relaxation times) dynamics near the wall, as measured by the stretching exponent $\beta$ of the KolrauschWilliam-Watts relaxation function. The average relaxation time decays towards the bulk value as one moves farther from the wall, with an exponential shape characterized by the dynamic penetration length $\xi_{d}$ (Fig. 15). At variance with the other results, the temperature dependence of $\xi_{d}$ shows a qualitative difference between the walls: while for PHY walls $\xi_{d}$ decreased with an increase in temperature, in the PHO case we found that the dynamic perturbation scale $\xi_{d}$ grows on heating. The qualitative difference can be appreciated graphically by plotting $\xi_{d}$ vs. $\xi_{s}$ (Fig. 16). Thus the answer to the question of whether a hydrophobic wall has more or less influence on water on changing the temperature depends on the property being considered: the structural influence decreases on heating (the static penetration length decreases), while the dynamic influence increases on heating (the dynamic correlation length grows).

\section{ACKNOWLEDGMENTS}

We thank J. R. Grigera and O. Chara for discussions and comments. C.G.F. was supported by Fundación Bunge y Born. This work was supported by grants from Consejo Nacional de Investigaciones Científicas y Técnicas (CONICET), Agencia Nacional de Promoción Científica y Tecnológica (ANPCyT), and Universidad Nacional de La Plata (UNLP, Argentina).

${ }^{1}$ M. Chaplin, Nat. Rev. 7, 861 (2006).

${ }^{2}$ P. Kumar, S. Han, and H. E. Stanley, J. Phys.: Condens. Matter 21, 504108 (2009).

${ }^{3}$ M. Erko, G. H. Findenegg, N. Cade, A. G. Michette, and O. Paris, Phys. Rev. B 84, 104205 (2011).

${ }^{4}$ K. Liu, Y. Zhang, J. Lee, C. Chen, Y. Yeh, S. Chen, and C. Mou, J. Chem. Phys. 139, 064502 (2013).

${ }^{5}$ G. Stirnemann, P. J. Rossky, J. T. Hynes, and D. Laage, Faraday Discuss. 146, 263 (2010).

${ }^{6}$ C. E. Bertrand, Y. Zang, and S. Chen, Phys. Chem. Chem. Phys. 15, 721 (2013).

${ }^{7}$ A. C. Fogarty, E. Duboue-Dijon, D. Laage, and W. H. Thompson, J. Chem. Phys. 141, 18C523 (2014).

${ }^{8}$ L. B. Krott and M. C. Barbosa, J. Chem. Phys. 138, 084505 (2013).

${ }^{9}$ E. B. Moore, J. T. Allen, and V. Molinero, J. Phys. Chem. 116, 7507 (2012).

${ }^{10}$ P. Kumar, F. W. Starr, S. V. Buldyrev, and H. E. Stanley, Phys. Rev. E 75, 011202 (2007)
${ }^{11}$ N. Giovambattista, P. J. Rossky, and P. G. Debenedetti, Phys. Rev. Lett. 102, 050603 (2009).

${ }^{12}$ N. Kastelowitz, J. C. Johnston, and V. Molinero, J. Chem. Phys. 132, 124511 (2010).

13 J. R. Grigera, S. G. Kalko, and J. Fischbarg, Langmuir 12, 154 (1996).

${ }^{14}$ S. Han, M. Y. Choi, P. Kumar, and H. E. Stanley, Nat. Phys. 6, 685 (2010).

${ }^{15}$ K. B. Jinesh and J. W. E. Frenken, Phys. Rev. Lett. 101, 036101 (2008).

${ }^{16}$ P. Gallo, M. Rovere, and E. Spohr, Phys. Rev. Lett. 85, 4317 (2000).

${ }^{17}$ P. Kumar, S. V. Buldyrev, F. W. Starr, N. Giovambattista, and H. E. Stanley, Phys. Rev. E 72, 051503 (2005).

${ }^{18}$ Y. Leng and P. T. Cummings, J. Chem. Phys. 124, 074711 (2006).

${ }^{19}$ H. Kumar, C. Dasgupta, and P. K. Maiti, RSC Adv. 5, 1893 (2015).

${ }^{20}$ B. A. Bauer, S. Ou, S. Patel, and K. Siva, Phys. Rev. E 85, 051506 (2012).

${ }^{21}$ H. J. C. Berendsen, J. R. Grigera, and T. P. Straatsma, J. Phys. Chem. 91, 6269 (1987)

${ }^{22}$ W. Kob and H. C. Andersen, Transp. Theory Stat. Phys. 24, 1179 (1995).

${ }^{23}$ B. Hess, C. Kutzner, D. van Der Spoel, and E. Lindahl, J. Chem. Theory Comput. 3, 435 (2008).

${ }^{24}$ H. J. C. Berendsen, J. P. M. Postma, A. DiNola, and J. R. Haak, J. Chem. Phys. 81, 3684 (1984).

${ }^{25}$ I. G. Tironi, R. Sperb, P. E. Smith, and W. F. van Gunsteren, J. Chem. Phys. 102, 5451 (1995).

${ }^{26}$ W. F. van Gunsteren and H. J. C. Berendsen, Gromos- 87 manual, Biomos BV Nijenborgh 4, 9747 AG Groningen, The Netherlands, 1987.

${ }^{27}$ A. R. van Buuren, S. J. Marrink, and H. J. C. Berendsen, J. Phys. Chem. 97, 9206 (1993).

${ }^{28}$ A. E. Mark, S. P. van Helden, P. E. Smith, L. H. M. Janssen, and W. F. van Gunsteren, J. Am. Chem. Soc. 116, 6293 (1994).

${ }^{29}$ W. L. Jorgensen, J. Chandrasekhar, J. D. Madura, R. W. Impey, and M. L. Klein, J. Chem. Phys. 79, 926 (1983).

${ }^{30}$ H. Liu, F. Müller-Plathe, and W. F. van Gunsteren, J. Am. Chem. Soc. 117, 4363 (1995).

${ }^{31}$ R. Kumar, J. R. Schmidt, and J. L. Skinner, J. Chem. Phys. 126, 204107 (2007).

${ }^{32}$ T. S. Grigera, V. Martín-Mayor, G. Parisi, and P. Verrocchio, Phys. Rev. B 70, 014202 (2004).

${ }^{33}$ N. Giovambattista, P. J. Rossky, and P. G. Debenedetti, J. Phys. Chem. B 113, 13723 (2009).

${ }^{34}$ A. A. Milischuk and B. M. Ladanyi, J. Chem. Phys. 135, 174709 (2011).

${ }^{35}$ M. U. Hammer, T. H. Anderson, A. Chaimovich, M. S. Shella, and J. Israelachvili, Faraday Discuss. 146, 299 (2010).

${ }^{36}$ O. Chara, A. N. McCarthy, C. G. Ferrara, E. Caffarena, and J. R. Grigera, Physica A 388, 4551 (2009).

${ }^{37}$ C. P. Lindsey and G. D. Patterson, J. Chem. Phys. 73, 3348 (1980).

${ }^{38}$ M. D. Ediger, C. A. Angell, and S. R. Nagel, J. Phys. Chem. 100(31), 13200 (1996).

${ }^{39}$ A. Shekhar, R. K. Kalia, A. Nakano, P. Vashishta, C. K. Alm, and A. Malthe-Sørenssen, Appl. Phys. Lett. 105, 161907 (2014).

${ }^{40}$ P. Scheidler, W. Kob, and K. Binder, J. Phys. Chem. B 108, 6673 (2004).

${ }^{41}$ N. Giovambattista, P. J. Rossky, and P. G. Debenedetti, Phys. Rev. E 73, 041604 (2006).

${ }^{42}$ L. B. Krott and M. C. Barbosa, Phys. Rev. E 89, 012110 (2014). 